\title{
Integrated analysis of microRNA and mRNA expression profiles in physiological myelopoiesis: role of hsa-mir-299-5p in CD34 + progenitor cells commitment
}

\author{
E Tenedini ${ }^{1,2}$, E Roncaglia ${ }^{1,2}$, F Ferrari $^{3}$, C Orlandi ${ }^{1}$, E Bianchi ${ }^{1}$, S Bicciato ${ }^{1,4}$, E Tagliafico $^{\star, 1,2,4}$ and S Ferrari ${ }^{1,2,4}$
}

Hematopoiesis entails a series of hierarchically organized events that proceed throughout cell specification and terminates with cell differentiation. Commitment needs the transcription factors' effort, which, in concert with microRNAs, drives cell fate and responds to promiscuous patterns of gene expression by turning on lineage-specific genes and repressing alternate lineage transcripts. We obtained microRNA profiles from human CD34 + hematopoietic progenitor cells and in vitro differentiated erythroblasts, megakaryoblasts, monoblasts and myeloblast precursors that we analyzed together with their gene expression profiles. The integrated analysis of microRNA-mRNA expression levels highlighted an inverse correlation between microRNAs specifically upregulated in one single-cell progeny and their putative target genes, which resulted in downregulation. Among the upregulated lineage-enriched microRNAs, hsa-miR-299-5p emerged as having a role in controlling CD34 + progenitor fate, grown in multilineage culture conditions. Gain- and loss-of-function experiments revealed that hsa-miR-299-5p participates in the regulation of hematopoietic progenitor fate, modulating megakaryocytic-granulocytic versus erythroid-monocytic differentiation.

Cell Death and Disease (2010) 1, e28; doi:10.1038/cddis.2010.5; published online 18 February 2010

Subject Category: Experimental Medicine

This is an open-access article distributed under the terms of the Creative Commons Attribution License, which permits distribution and reproduction in any medium, provided the original author and source are credited. This license does not permit commercial exploitation without specific permission.

Cell fate decisions in the hematopoietic system appear to be directed by an antagonistic or synergistic interplay of transcription factors that pivot immature blood progenitors for cell specification. ${ }^{1}$ Multipotent progenitors initially trigger a promiscuous transcriptional program, ${ }^{2,3}$ and as soon as they commit to a restricted fate, they reinforce unilineage gene expression and withdraw transcripts affiliated with alternative blood cell types. ${ }^{4,5}$ MicroRNAs appear to be especially pertinent in driving this particular behavior representing a new component of the hematopoietic gene regulatory network. ${ }^{6}$ In fact, the archetypal microRNA can potentially regulate hundreds of genes ${ }^{7-10}$ even if most targets contain isolated microRNA recognition sites that may be inadequate for complete gene silencing. According to Bartel's theory, ${ }^{11}$ microRNA-mediated post-transcriptional control offers a more flexible and rapid way of tuning genes compared with transcriptional control. These issues encouraged some investigators to explore the association of microRNAs and genes expression profiles obtained from the same cell type, ${ }^{7,12-14}$ and advocated that microRNAs evolved to regulate gene expression programs and remove gene products unnecessary or potentially dangerous more rapidly than might occur by natural decay. So far, these conclusions have not been fully substantiated in physiological myelopoiesis.

Although many studies addressed the role of microRNAs during the normal myeloid differentiation process, ${ }^{15-20}$ only Georgantas et al. ${ }^{21}$ focused on the impact of microRNAs on mRNA expression levels but limited the analyses to data obtained from human CD34 + stem/progenitor cells. ${ }^{21}$ To shed light on the interplay of mRNAs and microRNAs during the normal myeloid commitment and verify that increased expression of a microRNA is skillful in modulating the levels of corresponding target mRNAs, we obtained microRNA profiles from CD34 + hematopoietic progenitor cells (CD34 HPCs) and in vitro differentiated precursors: erythroblasts, megakaryoblasts, monoblasts and myeloblasts (ERY, MKC,

\footnotetext{
${ }^{1}$ Department of Biomedical Sciences, University of Modena and Reggio Emilia, Modena, Italy; ${ }^{2}$ BioPharmaNet, Emilia-Romagna High-Tech Network, Ferrara, Italy; ${ }^{3}$ Department of Biology, University of Padova, Padova, Italy and ${ }^{4}$ Center for Genome Research, University of Modena and Reggio Emilia, Modena, Italy ${ }^{*}$ Corresponding author: E Tagliafico, Department of Biomedical Sciences, Center for Genome Research, University of Modena and Reggio Emilia, Via Campi 287, Modena 41100, Italy. Tel: + 39059 2055387; Fax: + 0039059 2055410; E-mail: enrico.tagliafico@ unimore.it

Keywords: microRNA; microarrays; myelopoiesis; differentiation; integrated analysis

Abbreviations: CD34 HPCs, CD34 + hematopoietic progenitor cells; ERY, erythroblasts; MKC, megakaryoblasts; MYELO, myeloblasts; MONO, monoblasts; TPO, thrombopoietin; EPO, erythropoietin; RQ, relative quantity; GEO, gene expression omnibus database; SCF, stem cell factor; Gapdh, glyceraldehyde-3-phosphate dehydrogenase; FC, fold change; 299-5p, hsa-miR-299-5p pre-miR miRNA precursor molecule; NC1, pre-miR miRNA precursor molecule-negative control \# 1; anti-299-5p, hsa-miR-299-5p anti-miR miRNA inhibitor; anti-NC1, anti-miR miRNA inhibitor-negative control \#1; nuc, nucleofection solution-treated sample Received 07.12.09; accepted 14.1.10; Edited by R Mantovani
} 


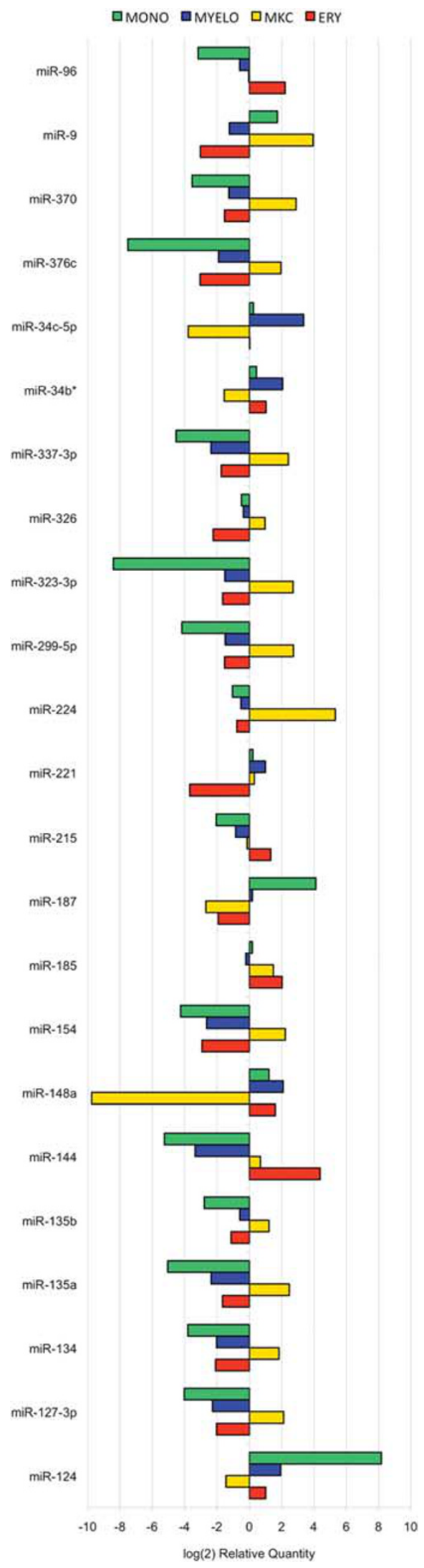

Cell Death and Disease
MONO and MYELO). We therefore analyzed these microRNA expression profiles together with the gene expression profiles of the same populations and observed that for the most part of the microRNAs specifically upregulated in one single progeny, an inverse correlation between microRNAs and downregulated putative target expression levels occurs, that is, downregulated genes showed an enrichment for the conserved putative targets of upregulated microRNA. Among these microRNAs, hsa-miR-299-5p emerged as an interesting candidate to show how the integrated analysis of microRNA and mRNA expression data can help shedding light on the regulatory mechanisms governing cell differentiation. In particular, we used hsa-miR-299-5p to prove that the forced expression of a single lineage-specific microRNA is able to control the cell fate of CD34 HPCs grown in multilineage culture conditions. Clonogenic and liquid culture differentiation assays after gain- and loss-of-function experiments revealed that indeed hsa-miR-299-5p regulates hematopoietic progenitor fate modulating megakaryocytic-granulocytic versus erythroid-monocytic development.

\section{Results}

Assessment of microRNA expression levels during physiological myeloid differentiation. We compared the expression profile of 156 mature microRNAs obtained by quantitative real-time PCR of each lineage precursor (ERY, MKC, MYELO and MONO) with those of CD34 HPCs as described in Materials and Methods section.

We selected those microRNAs whose expression was upor downregulated with a fold change greater than four in at least one of the four precursor populations when compared with the CD34 HPCs. When organized according to their expression patterns, 23 microRNAs resulted oppositely regulated, showing an upregulation in at least one precursor population and, concomitantly, a counter-regulation in at least another one (Figure 1).

MicroRNAs and mRNA expression integrated analysis. The integrated analysis of microRNAs and mRNA expression levels has been based on the assumption that increased or decreased expression of a specific microRNA results in, at least partially, decreased or increased expression of corresponding target mRNAs. Therefore, we investigated the existence of any inverse correlation between the up or downregulated expression of a micro-RNA and its predicted targets.

Figure 1 MicroRNAs oppositely regulated during myeloid differentiation. Twenty-three microRNAs (indicated with the prefix miR-) show either up- or downregulation greater than four in at least one precursor population when compared with the CD34 HPCs; 17 showed a significant increased expression with an $\mathrm{RQ}$ higher than four, 15 a significant decreased expression with an $\mathrm{RQ}$ lower than -4 and concomitantly a counter-regulation in at least one other precursor cell context. TaqMan real-time PCR reactions were carried out using the TaqMan MicroRNA Assays Human Panel Early Access Kit. Delta-delta-CTs and RQs were calculated for each detector using the CD34 HPC sample as the calibrator and hsamiR-let-7a as the endogenous control 
We compared microRNA and mRNA expression patterns (i) focusing on 17 microRNAs out of the 23 described above, which showed a significant increased expression (relative quantity, $R Q$, higher than four), and on the 15 microRNAs that showed a significant decreased expression (RQ lower than -4) in at least one precursor population and (ii) verifying if their 'highly conserved,' 'conserved' or 'human only' predicted target transcripts are enriched in counter-regulated genes $(P$-value of a hypergeometric test, see Materials and Methods section). The 15 downregulated microRNAs showed very few significant $P$-values (Supplementary Figure S1A-B), but a major part of the 17 upregulated microRNAs showed significant $P$-values when comparing each precursor with the CD34 HPCs (i.e., along with differentiation, 11 out of 17 were significant for at least one type of target), whereas a minor part were significant if compared with the other precursor populations (i.e., along with specificity, 6 out of 17 were significant) (Figure $2 a$ and $b$ ).

For each microRNA, the analysis was performed considering in separated tests the putative target mRNAs with different levels of conservation, according to TargetScan predictions: 'human only' targets, 'conserved' targets and 'highly conserved' targets were evaluated. The latter are supposed to be more reliable targets of a specific microRNA; nevertheless, human exclusive target genes may have a pertinent role in specific biological processes. No relevant
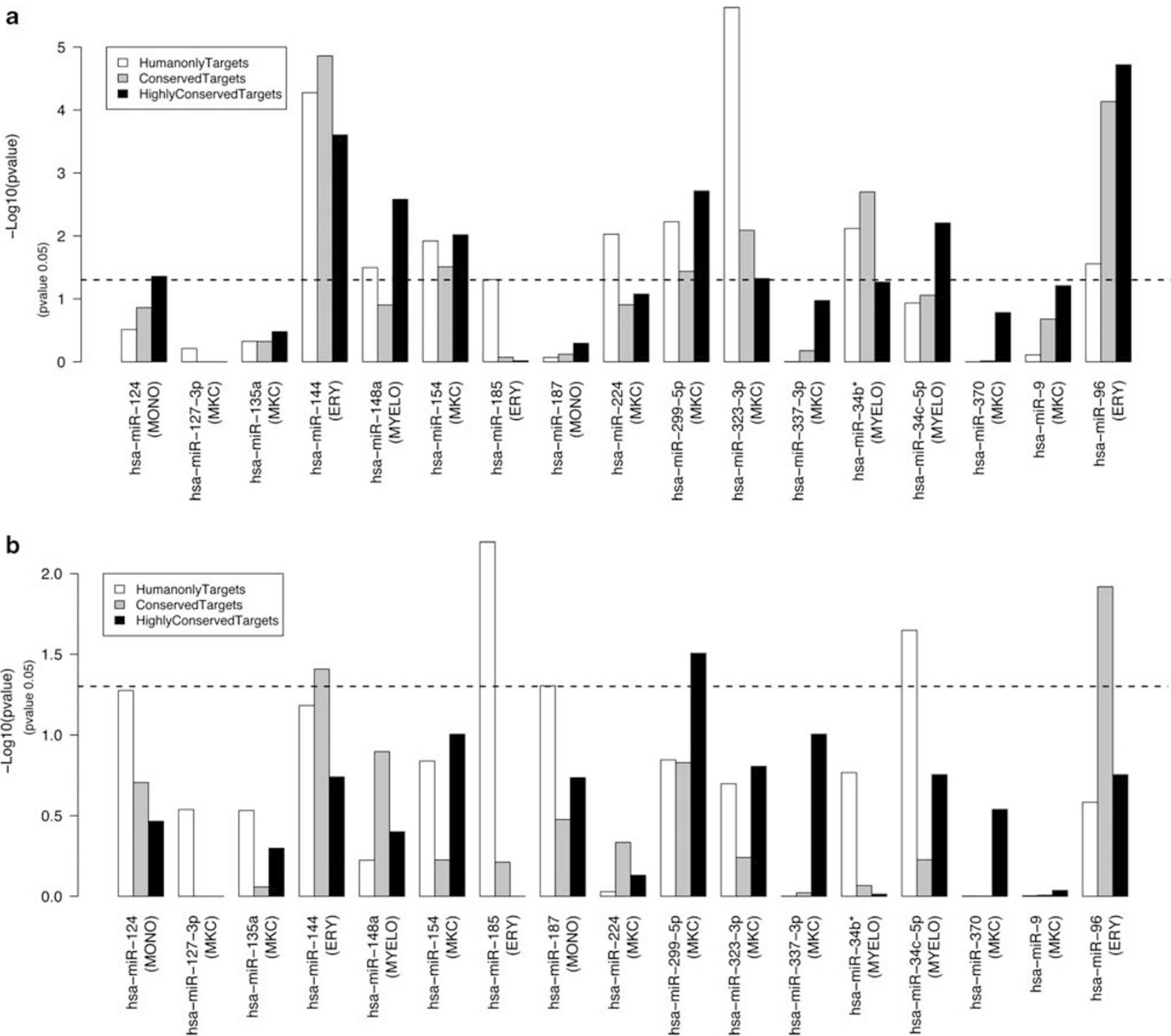

Figure 2 MicroRNA-mRNA expression-integrated analysis. MicroRNAs and target mRNAs expression patterns were analyzed for the 17 upregulated microRNAs in one specific precursor cell context. A hypergeometric test was used to test the enrichment in downregulated transcripts among their predicted targets: the analyses were performed considering in separated tests the putative target mRNAs with different levels of conservation ('highly conserved,' 'conserved' or 'human only' putative targets). Inverse logarithm of the obtained $P$-values is plotted as a bar plot and the reference precursor cell type for each microRNA is specified in the bar plot labels. A hypergeometric test result was considered significant if the $P$-value was equal to or lower than 0.05 . (a) $P$-values for the comparisons between precursor cells and CD34 HPCs; (b) $P$-values for the comparisons of each precursor cell context with the others 
4
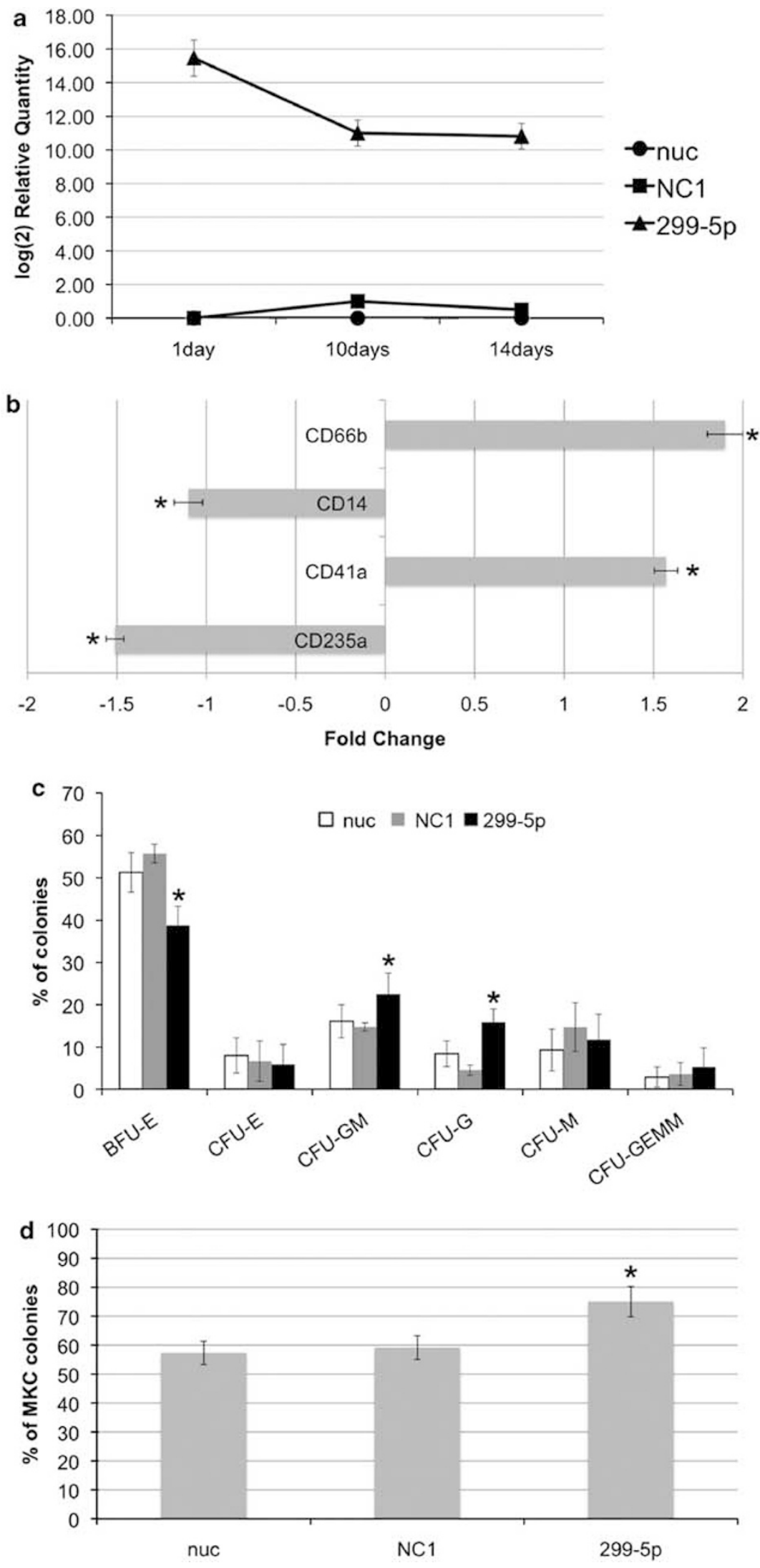
widespread differences emerged between 'highly conserved,' 'conserved' and 'human only' targets.

Hsa-miR-299-5p microRNA, found upregulated in MKC and downregulated in ERY, MYELO and MONO, showed a significant inverse correlation pattern (Figure $2 a$ and b). We chose this one as an exemplar microRNA to disclose the potential biological bearing of this kind of analytical approach.

The differential expression of hsa-miR-299-5p supports a role in CD34 HPC fate specification. We verified the expression levels of hsa-miR-299-5p microRNA in an independent set of human progenitors and CD34 HPC samples using TaqMan real-time PCR individual assay and no significant discrepancies were observed when compared with the results obtained with TaqMan microRNA Assays Human Panel Early Access Kit (Foster City, CA, USA) (Supplementary Figure S2).

To assess the hsa-miR-299-5p expression pattern throughout the studied myeloid differentiation pathways, a time course evaluation of hsa-miR-299-5p expression was monitored at $6,9,12$ and 15 days of in vitro MKC, ERY, MYELO or MONO differentiations of the same cord blood CD34 HPCs by means of TaqMan real-time PCR; cytofluorimetric analyses, and monitoring of CD235a, CD41a, CD14 and CD66b expression was performed alongside (see Materials and Methods section). The hsa-miR-299-5p expression increased quickly during MKC differentiation along with the appearance and the increase of surface antigen CD41a. On the other hand, the hsa-miR-299-5p microRNA showed no increase during ERY differentiation induction, in MYELO or MONO conditions, even if differentiation markers resulted induced (Supplementary Figure S3a-b). We can therefore consider hsa-miR-299-5p as a microRNA possibly involved in MKC differentiation.

Hsa-miR-299-5p changes the balance of
commitment. Hsa-miR-299-5p was further analyzed to
determine whether the forced expression of a single
lineage-enriched micro-RNA could control the cell fate of
CD34 HPCs grown in multilineage culture conditions.
Clonogenic assays and liquid culture differentiation allowed
us to quantitate the proportion of megakaryocytic, erythroid,
monocytic and granulocytic cells and to estimate if the
perturbation of one micro-RNA expression can change the
balance of the following commitment. Transient transfection
of hsa-miR-299-5p precursor molecule was performed
through a single nucleoporation with the Amaxa Biosystem

(Cologne, Germany) Nucleofector device into cord blood CD34 HPCs. The result was a consistent and rapid upregulation of the mature microRNA that was confirmed by TaqMan real-time PCR at different time points during in vitro culture of each cord blood sample (Figure 3a). MicroRNA forced expression biological effects were first examined by evaluating the expression of unilineage differentiation surface markers: CD235a that first appears at the basophilic normoblast stage of erythroid differentiation, ${ }^{22}$ CD41a as an indicator of megakaryocytic commitment, ${ }^{23}$ CD14 distinguishing monocytic differentiation ${ }^{24}$ while $\mathrm{CD}_{6} \mathrm{~b}^{25}$ reflects granulocytic cell fate. Hsa-miR-299-5p overexpression reduced the percentage of CD235a-positive cells (Figure $3 b$ ) and resulted in a decrease of erythroid burstforming unit (BFU-E) colonies in methylcellulose clonogenic assays (Figure 3c). It also diminished the number of erythrocytes in May Grunwald-Giemsa-stained cytospin preparations after 14 days of culture (Figure 4c and d). Besides, we observed an increase in CD41a expression, of megakaryocytic colonies in collagen-based assays (Figure $3 b-d$ ) and also of MKC forms with their characteristic polynucleated elements in morphologies (Figure $4 \mathrm{c}$ and $\mathrm{d}$ ). Moreover, we reported modifications in the expression of granulocyte and monocyte markers and in the relative abundance of grown colonies: the common trend consisted in an increase of the granulocytic tracts and in a drop of monocytic ones (Figures $3 \mathrm{~b}, \mathrm{c}$ and $4 c$ and d).

To complete these miR-299-5p forced expression studies, we turned to a loss-of-function method. CD34 HPCs were isolated and cultured for $72 \mathrm{~h}$ in the presence of thrombopoietin (TPO) to assure the upregulation of the mature hsamiR-299-5p expression (Figure 5a). Then, we assayed them for colony formation in methylcellulose and collagen in the presence or absence of a 299-5p anti-miRNA inhibitor (see Materials and Methods section). Hsa-miR-299-5p anti-miR miRNA inhibitor (anti-299-5p) but not the anti-miR-negative control 1 (anti-NC1) fully knocked down miR299-5p expression after $24 \mathrm{~h}$ (Figure $5 \mathrm{~b}$ ). Treated samples with anti-299-5p showed a decrease in CFU-Mk and colony-forming units granulocyte (CFU-G) colonies, compared with anti-NC1 (Figure $5 \mathrm{c}$ and $\mathrm{d}$ ), and a proportional increase in BFU-E (Figure 5c).

To corroborate these observations, $24 \mathrm{~h}$ after hsa-miR-299$5 p$ forced expression, we evaluated some hematopoietic differentiation markers by means of TaqMan real-time PCR and measured a decrease in the erythroid-specific messengers (Rh-associated glycoprotein, Rhag; hemoglobin alpha 1, Hba1 and Kruppel-like factor 1, KIf-1) and an increase in the

Figure 3 Pre-miR miRNA Precursor Molecule transfections. (a) TaqMan real-time PCR of mature hsa-miR-299-5p expression after single transient transfection of its precursor molecule through the Amaxa Nucleofector device. Hsa-miR-299-5p expression was monitored at different time points during in vitro culture using the nucleofection solution-treated sample (nuc) as calibrator and RNU6b as endogenous control. (b) Cytofluorimetric evaluation of the differentiation surface markers' expression: CD235a for erythroid differentiation, CD41a as indicator of megakaryocytic commitment, CD14 distinguishing monocytic differentiation and CD66b reflecting granulocytic cell fate. The surface markers' expression was checked after seven days of culture and each bar represents the fold change of the percentage of positivity normalized onto the pre-miR miRNA precursor molecule-negative control \# 1 (NC1)-treated sample. Asterisk indicates a $t$-test $P$-value $\leqslant 0.001$. (c) Colony-forming capacity of HPCs transfected with premiR miRNA precursor molecule (299-5p), when compared with Pre-miR miRNA Precursor molecule-negative control \# 1 (NC1)-transfected cells and with cells electroporated with the nucleofection solution only (nuc). Mean \pm S.D. from four independent experiments. BFU-E: erythrocyte burst-forming unit; CFU-E: erythrocyte colony-forming unit; GM-CFU: granulo-monocyte colony-forming unit; G-CFU: granulocyte colony-forming unit; M-CFU: monocyte colony-forming unit; GEMM-CFU: granulocyte-erythrocytemonocyte-macrophage colony-forming unit. Asterisk indicates a $t$-test $P$-value $\leqslant 0.001$. (d) Megakaryocytic colony-forming capacity of HPCs transfected with pre-miR miRNA precursor molecule (299-5p), when compared with pre-miR miRNA precursor molecule-negative control \# 1 (NC1)-transfected cells and cells electroporated with the nucleofection solution only (nuc). Mean \pm S.D. from four independent experiments. Asterisk indicates a $t$-test $P$-value $\leqslant 0.001$ 
granulocytic markers myeloperoxidase (Mpo) and elastase 2 (Ela2) together with the megakaryocytic-determining factor friend leukemia virus integration 1 (Fli1) (Figure 6).

\section{Discussion}

Hematopoiesis involves a series of hierarchically organized events that proceed throughout cell specification and end with cell differentiation. Commitment needs the transcription factors' effort, which, together with microRNAs, pivots cell fate specification, answering to promiscuous patterns of gene expression by turning on lineage-specific genes and repressing alternate lineage transcripts. ${ }^{1}$ Therefore, microRNAs and mRNAs seem to cooperate to direct cell fate hematopoietic decisions. To date, those studies that addressed the process of human hematopoietic commitment focused on one or two differentiation pathways and on single or very few microRNAs ${ }^{15-18,20,21,26-29}$ without attempting to explore any extensive correlation between mRNA and microRNA expression profiles in multiple samples.

We decided, therefore, to proceed to analyze different hematopoietic precursors simultaneously (ERY, MKC, MYELO and MONO) with the leading hypothesis that increased expression of a cell-context-enriched microRNA is believed to result in at least partial decreased expression of matching target mRNAs.

Given the limited number of samples, we excluded the application of the GenMiR ++ algorithm ${ }^{30}$ for the analysis of our data set. Indeed, although being a Bayesian method specifically proposed for refining the prediction of miRNA targets through the combination of paired miRNA and mRNA expression profiles, GenMiR + + cannot guarantee robust results for small data sets.
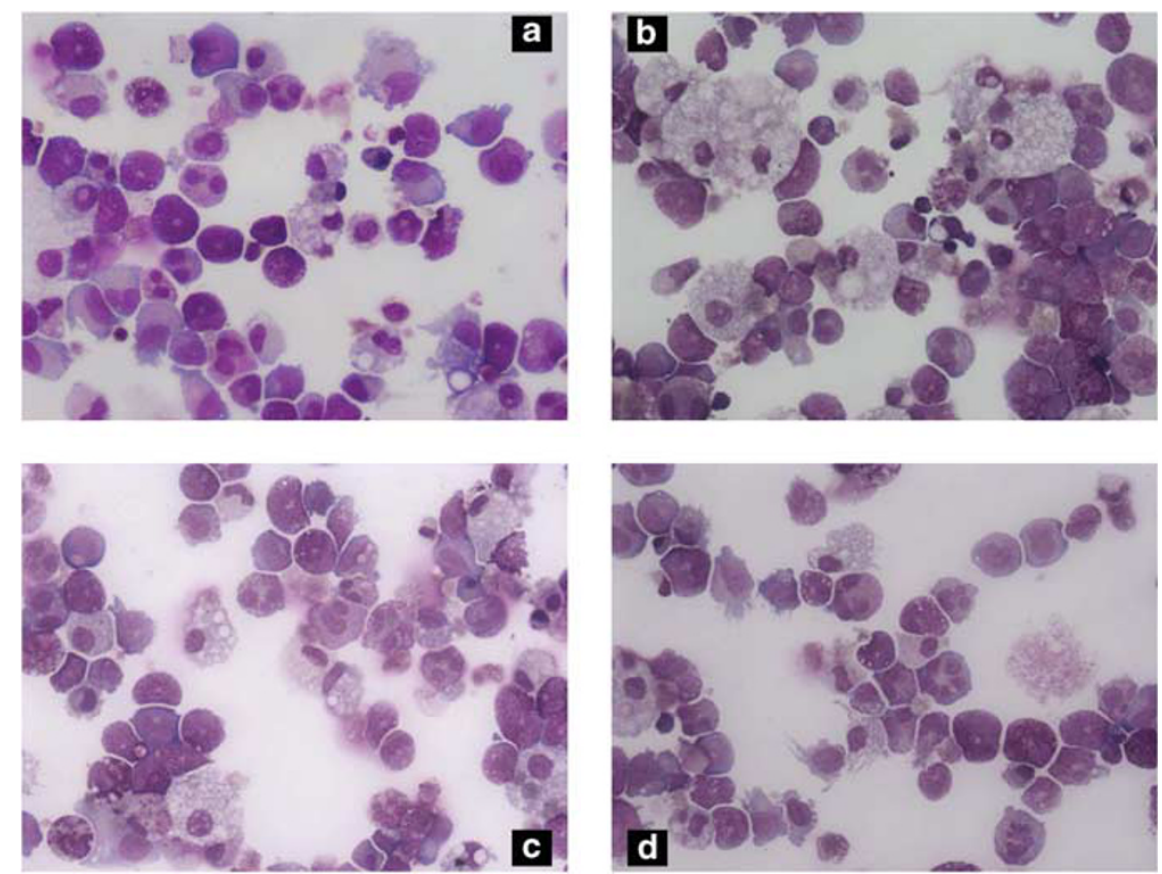

Figure 4 Morphologic analysis after pre-miR miRNA precursor molecule transfections. May-Grunwald staining ( $\times 20$ magnification, 0.5 aperture objective lenses (Carl Zeiss)) of cytospin preparations after 14 days of culture in a representative experiment. Morphology was evaluated with an Axioskop 40 microscope (Carl Zeiss, Oberkochen, Germany). The camera was inserted within the microscope. No software was used to optimize the figure. Objective lenses $\times 20$ and $\times 40$. (a) and (b) Precursor moleculenegative control \# 1-treated cells. (c) and (d) Hsa-miR-299-5p pre-miR miRNA precursor molecule-treated cells

Figure 5 Anti-miRNA inhibitor molecule transfections. (a) TaqMan real-time PCR evaluation of mature hsa-miR-299-5p microRNA expression after 24, 72 and $96 \mathrm{~h}$ of cell culture in the presence of TPO $(100 \mathrm{ng} / \mathrm{ml})$. Data were obtained using as calibrator the RNA of the same sample extracted immediately after CD34 HPC purification and RNU6b as endogenous control. Cells were electroporated with hsa-miR-299-5p inhibitor after 3 days of culture. (b) TaqMan real-time PCR mature hsa-miR-299-5p expression after single transient transfection of its inhibitor molecule, through single nucleoporation with the Amaxa Nucleofector device. Expression withdrawal was monitored after one and ten days from nucleofection. Data were obtained using as calibrator the nucleofection solution-treated sample (nuc) and RNU6b as endogenous control. (c) Colonyforming capacity of HPCs transfected with hsa-miR-299-5p Anti-miR miRNA inhibitor (anti-299-5p), compared with Anti-miR miRNA inhibitor-negative control \#1 (anti-NC1)transfected cells and cells electroporated with the nucleofection solution only (nuc). Mean \pm S.D. from three independent experiments. Asterisk indicates a $t$-test $P$-value $\leqslant 0.001$. BFU-E: erythrocyte burst-forming unit; CFU-E: erythrocyte colony-forming unit; GM-CFU: granulo-monocyte colony-forming unit; G-CFU: granulocyte colonyforming unit; M-CFU: monocyte colony-forming unit; GEMM-CFU: granulocyte-erythrocyte-monocyte-macrophage colony-forming unit. (d) Megakaryocytic colony-forming capacity of HPCs transfected with hsa-miR-299-5p Anti-miR miRNA Inhibitor (anti-299-5p), compared with anti-miR miRNA inhibitor-negative control \#1 (anti-NC1)-transfected cells and cells electroporated with the nucleofection solution only (nuc). Mean \pm S.D. from three independent experiments. Asterisk indicates a $t$-test $P$-value $\leqslant 0.001$ 


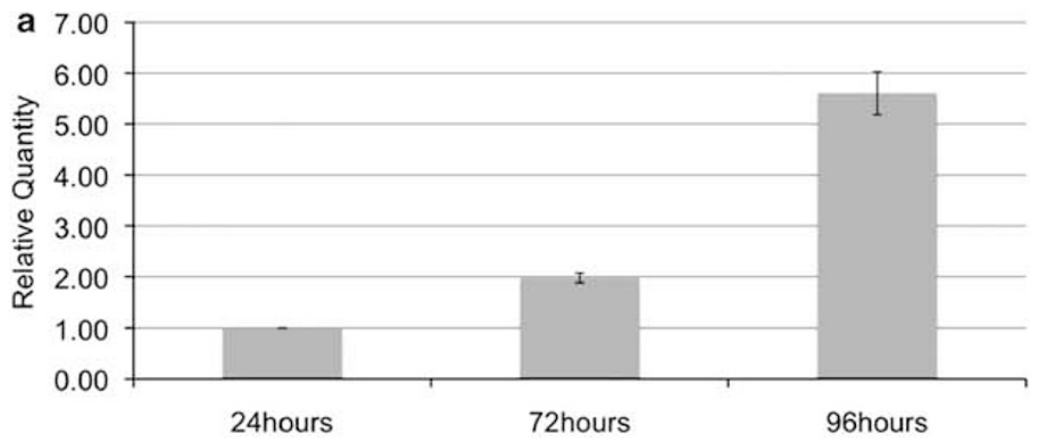

b

$\square$ nuc $=$ anti-NC1 $=$ anti-299-5p

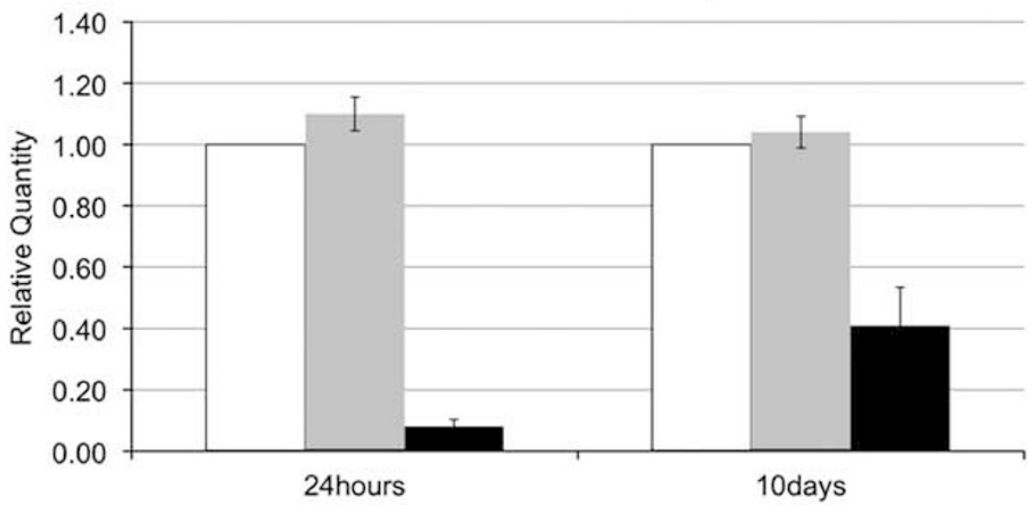

C

口nuc = anti-NC1 $=$ anti-299-5p
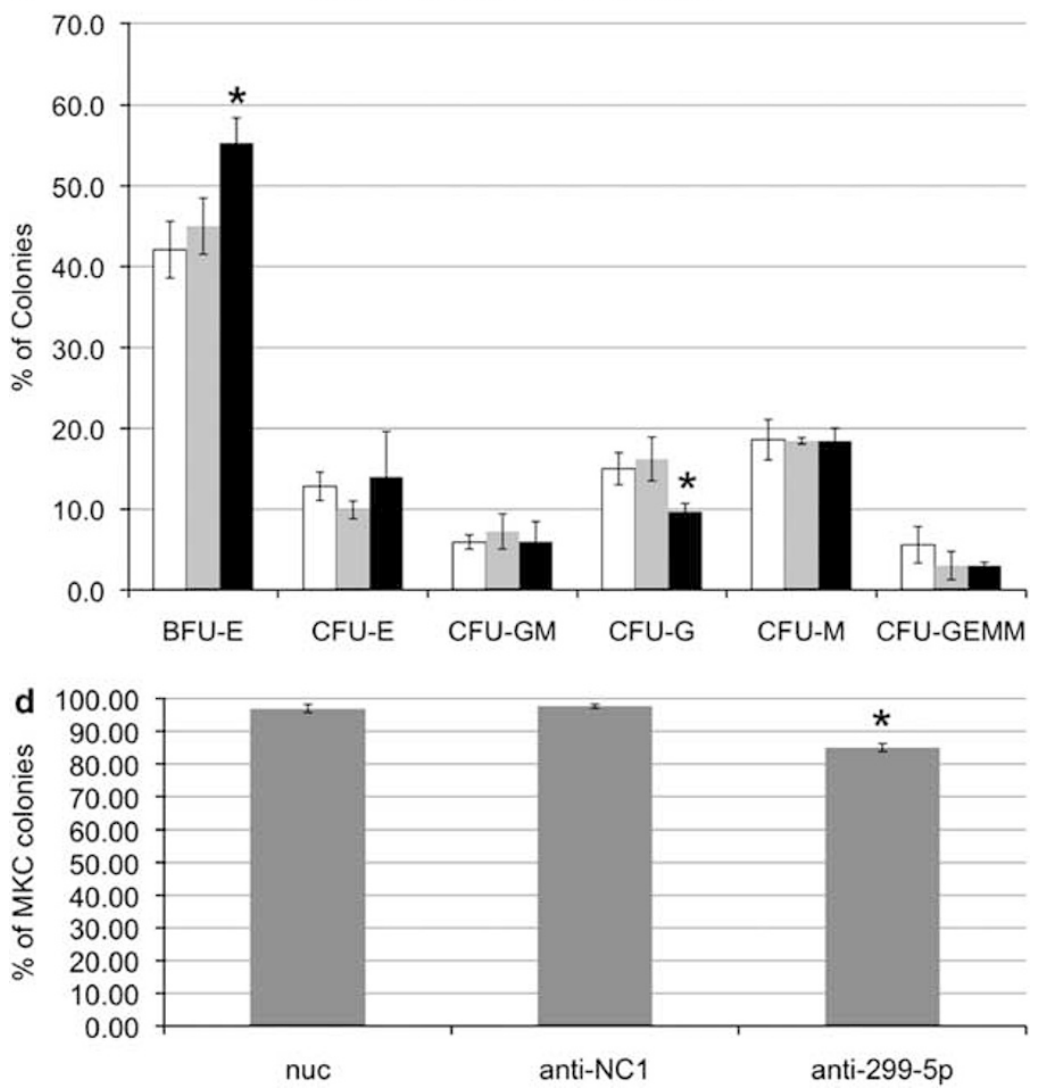


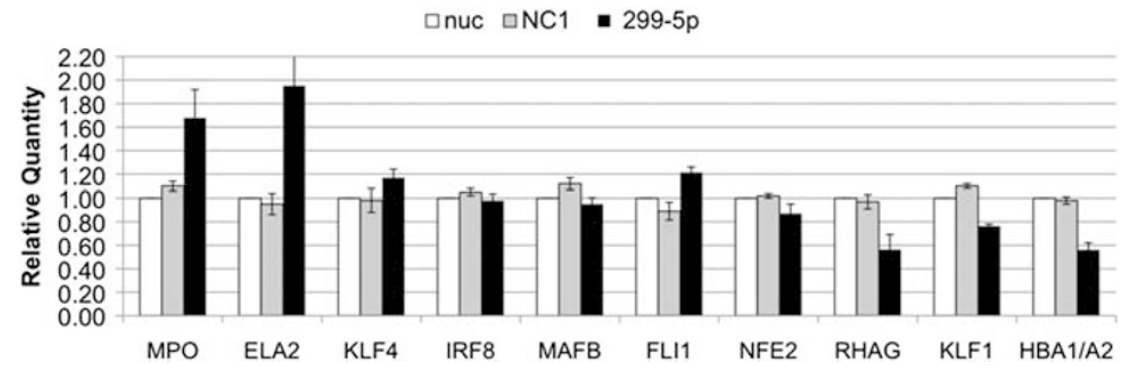

Figure 6 Evaluation of the myeloid differentiation markers' expression by quantitative real-time PCR. The figure shows the decrease of the erythrocyte plasma membrane glycoprotein Rh-associated glycoprotein, Rhag; hemoglobin alpha 1, Hba1 and of the Beta-globin gene expression regulator, Kruppel-like factor 1(Klf1). Alongside, it shows the increase in the granulocytic markers myeloperoxidase $(\mathrm{Mpo})$ and elastase 2 (Ela2) together with the megakaryocytic-determining factor friend leukemia virus integration 1 (Fli1). Nuclear factor (erythroid-derived 2), Nfe2, which is involved in the late differentiation phase of platelet assembly and release, as expected, was not significantly upregulated and neither were the monocytic markers interferon regulatory factor 8 (IIf8), Kruppel-like factor 4 (Klf4) and v-Maf musculoaponeurotic fibrosarcoma oncogene homolog B (Mafb). Delta-delta-CTs and RQs were calculated for each detector using as calibrator the nucleofection solution-treated sample (nuc) and glyceraldehyde-3phosphate dehydrogenase as endogenous control. Mean \pm S.D. from three independent experiments

Hypergeometric tests confirmed that target transcripts of many upregulated hematopoietic microRNAs are enriched in specific downregulated genes. This test represents a useful indication, able to suggest the biological relevance of a specific microRNA in shaping from a common CD34+ progenitor a peculiar and specialized hematopoietic precursor and interfering with the differentiation processes throughout its target genes. Furthermore, this kind of approach returns hints of a relationship statistically significant and completely regardless of the established and/or putative role of target mRNAs in myelopoiesis.

To confirm this postulation, we decided to provide further insights into microRNA's biological role by studying the potential unbalancing effects of a single microRNA during the commitment of human CD34 + hematopoietic stem/ progenitor cells. Many studies showed that the change in the expression of a single microRNA can reinforce single lineage differentiation pathway into already committed cells or into progenitors grown in unilineage conditions. ${ }^{15-17,19,31}$ Lu et al. ${ }^{18}$ recently assessed a causal role of miR-150 in the specification of megakaryocyte versus erythrocyte, when using a bilineage primary cell culture system, where human bone marrow $\mathrm{CD} 34+$ hematopoietic progenitors were cultured in the presence of TPO and erythropoietin (EPO) concomitantly. We asked instead if the ectopic expression of a single lineage-specific micro-RNA could control the cell fate of CD34 + progenitors grown in multilineage conditions. This method, testing if perturbation of one micro-RNA expression can change the balance of the following commitment, allowed us to estimate the proportion of all the four myeloid populations. We chose hsa-miR-299-5p, whose microRNAmRNA's inverse correlation was significant, particularly when considering 'highly conserved' putative targets. Hsa-miR-299$5 p$ overexpression in CD34 HPCs resulted to influence the hematopoietic commitment. Moderate but consistent inverse consequences of overexpression and knockdown experiments and coherence with gene expression profiling data let us conclude that hsa-miR-299-5p exerts its action of support to the megakaryocytic pathway by impairing mostly the erythroid precursors and preventing the block of granulocytic differentiation.
In summary, we tested the existence of a close relationship between microRNA and mRNA expression profiles during hematopoietic physiological commitment. We established significant inverse correlations between some specifically upregulated microRNAs and their putative mRNAs target levels. We chose one micro-RNA from this selection and verified its role in CD34 HPC commitment.

\section{Materials and Methods}

CD34 + Hematopoietic progenitor cells (CD34 HPCs) purification and liquid suspension cultures. Human CD34 HPC cells were purified from umbilical cord blood samples, and collected anonymously according to the institutional guidelines for discarded material and the Helsinki declaration. Mononuclear cells were isolated using the EasySep Human Whole Blood/Buffy Coat CD34-Positive Selection Kit (StemCell Technologies, Vancouver, Canada) following the manufacturer's instructions. Samples yielding a purity lower than $95 \%$ were discarded.

MYELO and MONO were obtained from CD34 HPC cells as previously described ${ }^{32}$ Briefly, they were obtained by plating CD34 HPCs at a density of $0.5 \times 10^{6} \mathrm{cell} / \mathrm{s} / \mathrm{ml}$ in Iscove's Modified Dulbecco's Medium (IMDM) (GIBCO, Grand Island, NY, USA) supplemented with 20\% FBS (Bio-Whittaker, Walkersville, MD, USA), $2 \mathrm{mM} \mathrm{L-glutamine,} 100 \mu \mathrm{g} / \mathrm{ml}$ streptomycin, $100 \mathrm{U} / \mathrm{ml}$ penicillin, with the addition of $50 \mathrm{ng} / \mathrm{ml}$ stem cell factor (SCF), $50 \mathrm{ng} / \mathrm{ml}$ FMS-like tyrosine kinase 3 ligand, $10 \mathrm{ng} / \mathrm{ml}$ interleukin- 6 and $10 \mathrm{ng} / \mathrm{ml}$ interleukin-3 (all from R\&D Systems, Minneapolis, MN, USA). Cells were monitored by flow cytometry for the expression of CD14 and purified after 8 days of culture using the EasySep Human CD14Positive Selection kit (StemCell Technologies) that yielded a purity of $96.7 \%$ (S.D. $\pm 2.4, n=5$ ).

Human MKCs were obtained, as formerly illustrated, ${ }^{33}$ by plating CD34 HPC progenitors at a density of $0.1 \times 10^{6} \mathrm{cells} / \mathrm{ml}$ in IMDM (GIBCO) supplemented with $20 \%$ bovine serum albumin, insulin and transferrin (BIT) serum substitute (StemCell Technologies), $1 \mathrm{mM}$ L-glutamine, $100 \mu \mathrm{g} / \mathrm{ml}$ streptomycin, $100 \mathrm{U} / \mathrm{ml}$ penicillin, with the addition of $100 \mathrm{ng} / \mathrm{ml}$ human recombinant TPO (R\&D Systems) every 3 days for 14 days. After 12-14 days of culture, MKC cells were purified using the EasySep Do-It-Yourself Positive Selection kit (StemCell Technologies) and anti-CD41a monoclonal antibody (Becton Dickinson, San Jose, CA, USA) with a median yield of $97.5 \%$ (S.D. $\pm 1.9, n=5$ ).

Human normal ERYs were obtained as previously explained ${ }^{34}$ by plating CD34 HPCs at a density of $0.5 \times 10^{6} \mathrm{cell} / \mathrm{s} / \mathrm{ml}$ in IMDM supplemented with $20 \%$ BIT (StemCell Technologies), $50 \mathrm{ng} / \mathrm{ml} \mathrm{SCF}$ and $4 \mathrm{U} / \mathrm{ml}$ EPO (R\&D Systems). Cells were monitored for the expression of surface antigen CD235a every 3 days of liquid culture and EPO was added. After 12-14 days of culture, cells were collected and the purity assessed (92\% CD235a-positive cells, S.D. $\pm 2.33, n=5$ ). Purity of CD34 HPCs and precursor cell populations was also assessed with morphological analysis, using MayGrunwald-Giemsa staining. 
MicroRNA expression profiles. Total cellular RNA was extracted from five CD34 HPCs, MYELO, MONO, MKC and ERY populations using the miRVana miRNA Isolation kit (Life Technologies-Ambion, Austin, TX, USA) following the manufacturer's protocol. The quantity and purity/integrity of each sample was quantified using Nanodrop 1000 (Thermo Fisher Scientific, Freemont, CA, USA) and 2100 Bioanalyzer (Agilent Technologies, Palo Alto, CA, USA). Five RNAs for each cellular context were then pooled and checked again for purity and quantity.

Total RNAs were reverse transcribed and TaqMan PCR reactions were carried out using the TaqMan MicroRNA Assays Human Panel Early Access Kit (Life Technologies-Applied Biosystems, Foster City, CA, USA). PCR reactions were performed using TaqMan Universal PCR Master Mix without AmpErase Uracil $\mathrm{N}$ glycosylase, by means of the ABI PRISM $7900 \mathrm{HT}$ Sequence Detection Systems (all from Life Technologies-Applied Biosystems). For each pooled sample (CD34 HPCs, MYELO, MONO, MKC and ERY), we obtained data from the two 96-well plates containing the microRNA TaqMan Assays (PlateA and PlateB). We performed three replicates for each of the two plates (Rep1, Rep2 and Rep3), and therefore the data set comprises a total of 30 ninety-six-well plates.

Statistical analyses were obtained using the 2-Delta-delta-Cycle Threshold values (Delta-delta-CTs) method ${ }^{35}$ and the Threshold was determined automatically by means of SDS Enterprise Edition software (Life Technologies-Applied Biosystems). Delta-delta-CTs and $\mathrm{RQ}$ were calculated for each detector using the CD34 HPCs sample as a calibrator and hsa-miR-let-7a as the endogenous control (average CT for the five samples equal to 26.19; S.D. $=0.43$ ).

All those microRNAs whose expression turned out to be undetermined or that showed cycle threshold values higher than 34 in all populations, that is, 'not expressed' in our data set, were filtered out. MicroRNA expression data are available in the gene expression omnibus (GEO) database with accession number GSE17586 (http://www.ncbi.nih.gov/geo).

mRNA expression profiles. cDNA synthesis, biotin-labeled target synthesis, HG-U133A GeneChip arrays hybridization, staining and scanning were performed according to the GeneChip One-Cycle Target Labeling and Control Reagents protocol supplied by the manufacturer (Affymetrix, Santa Clara, CA, USA).

Human myeloid data set. The data set of myeloid gene expression profiles covered both CD34 HPCs and myeloid precursor cells (ERY, MYELO, MONO, $M K C)$. Raw data were obtained, processed and annotated as previously described ${ }^{36}$ so as to remove probes with ambiguous matches to obtain gene expression data with reliable biological annotations. Raw expression data files for the human myeloid data set were previously published in the GEO database with accession number GSE12803. The normalized data table obtained as already described $^{36}$ is also provided as Supplementary material (Supplementary Table S1). Median expression level is reported for each considered cell type that was used for the integrated analysis of microRNA and mRNA expression profiles.

Integrated analysis of microRNA and mRNA expression profiles. For each microRNA with increased expression in a specific precursor cell type, putative targets were selected considering different conservation criteria as described below. Then, for each microRNA with increased expression in specific precursor cell types, a hypergeometric test was used to verify whether putative target transcripts are enriched in downregulated genes, or alternatively, if they are enriched in genes with the lowest expression in that specific precursor cell context. Conversely, the same approach was applied for downregulated microRNAs evaluating the potential enrichment in putative target genes among upregulated mRNAs. The genes were classified as downregulated or upregulated in a specific precursor cell type, if they showed lower or higher median expression level, respectively, in the specific precursor when compared with median expression level in CD34 HPCs and if they showed the lowest or the highest expression in a specific precursor cell context, when compared with the other precursors.

Hypergeometric test was implemented in $\mathrm{R}$ software and its result was considered significant if the $P$-value was equal to or lower than 0.05

\section{MicroRNA putative target selection. TARGETSCAN} (www.targetscan.com) version 4.1 was used to select putative target mRNAs for each individual differentially expressed microRNA. We focused on putative targets with different degrees of conservation across various organisms. The definition of conserved and non-conserved putative mRNA targets is based on the identification of specific microRNA binding sites in each mRNA across different species, according to TARGETSCAN 4.1 predictions. In particular, for each microRNA considered, a list of 'conserved target' mRNAs was defined by selecting mRNAs with a specific microRNA binding site conserved in humans, mice, rats and dogs. Then, a list of 'human only targets' was defined by selecting transcripts with a specific microRNA binding site that is present only in the human mRNA. Moreover, for additional analyses, the lists of 'highly conserved targets' were defined as mRNAs with a specific binding site conserved both in mammals (humans, mice, rats, dogs) and in chickens.

\section{Pre-miR miRNA precursor molecule and Anti-miRNA inhibitor transfections}

Pre-miR miRNA precursor molecule transfections. After separation, CD34HPCs were plated at the same conditions as previously described. ${ }^{37}$ After 2 days of culture, they were transfected with the Amaxa Nucleofector Device, using the Human CD34 Cell Nucleofection Kit, according to the manufacturer's instructions (Amaxa Biosystem), and $5 \mu \mathrm{g}$ of either the pre-miR miRNA precursor molecule-negative control \# 1 (NC1) or the hsa-miR-299-5p pre-miR miRNA precursor molecule (Ambion, Austin, TX, USA) and pulsed with the program U-008.

Pre-miR negative control 1 is a random sequence pre-miR molecule that has been extensively tested in human cell lines and tissues and validated to not produce identifiable effects on known miRNA function (http://www.ambion.com). To further exclude nucleofection-aspecific effects, one sample was electroporated with the nucleofection solution only (nuc).

Anti-miRNA inhibitor transfections. After separation, CD34 HPCs were plated in IMDM (GIBCO), 20\% BIT serum substitute (StemCell Technologies) and TPO (100 ng/ml) (R\&D Systems). After 3 days of culture, they were transfected with $5 \mu \mathrm{g}$ of either the anti-NC1 or the anti-299-5p (Ambion) and pulsed with the program $\mathrm{U}-008$. Anti-miR negative control \#1 is a random sequence anti-miR molecule that has been extensively tested in human cell lines and tissues and validated to not produce identifiable effects on known miRNA function (http://www.ambion.com). To further exclude nucleofection-aspecific effects, one sample was electroporated with the nucleofection solution only (nuc)

After $24 \mathrm{~h}$ from either the hsa-miR-299-5p precursor or anti-miR nucleofections, cells were plated following the manufacturer's instructions in MethoCult GF H4434 complete methylcellulose medium for clonogenic assay (StemCell Technologies) containing a cocktail of recombinant human cytokines: SCF $(50 \mathrm{ng} / \mathrm{ml})$, granulocytemacrophage colony-stimulating factor $(10 \mathrm{ng} / \mathrm{ml})$, interleukin-3 $(10 \mathrm{ng} / \mathrm{ml})$ and EPO $(3 \mathrm{U} / \mathrm{ml})$. After 14 days of culture at $37^{\circ} \mathrm{C}$ in a humidified atmosphere with $5 \% \mathrm{CO}_{2}$, colonies were scored as BFU-Es, erythroid colony-forming units (CFU-E), CFU-G, macrophage (CFU-M), granulocyte-macrophage and colony-forming units granulocyte/erythrocyte/macrophage/megakaryocyte.

After $24 \mathrm{~h}$ from either the hsa-miR-299-5p precursor or anti-miR nucleofections, cells were plated following the manufacturer's instructions in MegaCult-C (StemCell Technologies) for megakaryocytic assay. CD34 HPCs were cultured at a density of $2.5 \times 10^{3} / \mathrm{ml}$ in a collagen-based medium and a $0.75 \mathrm{ml}$ cell suspension was seeded per chamber of a double-chamber slide. This collagen-based system contains a medium supplemented to a final concentration with $1.1 \mathrm{mg} / \mathrm{ml}$ collagen, $1 \%$ bovine serum albumin, $0.01 \mathrm{mg} / \mathrm{ml}$ bovine pancreatic insulin, $0.2 \mathrm{mg} / \mathrm{ml}$ human iron-saturated transferrin and the human recombinant cytokines $50 \mathrm{ng} / \mathrm{ml} \mathrm{TPO}$, $10 \mathrm{ng} / \mathrm{ml}$ interleukin-3 and $10 \mathrm{ng} / \mathrm{ml}$ interleukin-6. Chamber slides were incubated at $37^{\circ} \mathrm{C}$ for 12 days and then fixed for $20 \mathrm{~min}$ in 1:3 methanol/acetone. Megakaryocyte colonies were stained using a primary monoclonal anti-CD41 antibody and then identified using an alkaline phosphatase detection system (all from StemCell Technologies). Nuclei of all the cells regardless of lineage were counterstained with Evans Blue. CD41-positive colonies were scored as CFU-Mk.

CD34HPCs were cultured and differentiation was monitored by morphological analysis and by flow cytometric analysis of CD14, CD66b, CD235a and CD41a surface antigen expression (Becton Dickinson), as previously described. ${ }^{37}$

Real-time PCR. Total cellular RNA was extracted at different time points using the miRVana miRNA Isolation Kit (Ambion) starting $24 \mathrm{~h}$ after nucleofection of either the hsa-miR-299-5p precursor or the anti-miR molecule, and the expression of the hsa-miR-299-5p mature microRNA was evaluated by means of the single real-time PCR TaqMan MicroRNA Assay (Life Technologies-Applied Biosystems). Deltadelta-CTs and $\mathrm{RQ}$ were calculated for each detector using as calibrator the nuc sample and the TaqMan Control miRNA Assay RNU6b as endogenous control.

Hematopoietic lineage-specific messenger evaluation was assessed using TaqMan Gene Expression Assays and TaqMan Universal PCR Master Mix (Life Technologies-Applied Biosystems). Delta-delta-CTs and RQ were calculated for each detector using as calibrator the nuc sample and glyceraldehyde-3-phosphate dehydrogenase as endogenous control. 


\section{Conflict of interest}

The authors declare no conflict of interest.

Acknowledgements. We are grateful to Dr Marisa De Palma and Dr Chiara Carboni (Transfusion Service and Blood Bank of Policlinico Hospital, Modena, Italy) for collection of umbilical cord blood samples. This work was supported by grants from AIRC (Italian Association for Cancer Research) regional grant (E Tagliafico), Italian PRIN (Italian Research Projects of National Relevance) (S Ferrari), AIRC Oncogenomics grant (E Tagliafico and S Ferrari), CIB (Italian interuniversity Biotecnology Consortium) and AIBG (Italian Association of Biology and Genetics). Elena Tenedini is a BioPharmaNet fellow (http://www.biopharmanet.eu) and this work is in partial fulfillment of the requirement for the BioPharmaNet Genomics and Biosensors research projects. This work is dedicated to the memory of our colleague and friend Stefano Ferrari.

1. Laslo $P$, Pongubala JM, Lancki DW, Singh $\mathrm{H}$. Gene regulatory networks directing myeloid and lymphoid cell fates within the immune system. Semin Immunol 2008; 20: 228-235.

2. Manfredini R, Zini R, Salati S, Siena M, Tenedini E, Tagliafico E et al. The kinetic status of hematopoietic stem cell subpopulations underlies a differential expression of genes involved in self-renewal, commitment, and engraftment. Stem Cells 2005; 23: 496-506.

3. Rothenberg EV. Stepwise specification of lymphocyte developmental lineages. Curr Opin Genet Dev 2000; 10: 370-379

4. Hu M, Krause D, Greaves M, Sharkis S, Dexter M, Heyworth C et al. Multilineage gene expression precedes commitment in the hemopoietic system. Genes Dev 1997; 11: 774-785.

5. Zhu J, Emerson SG. Hematopoietic cytokines, transcription factors and lineage commitment. Oncogene 2002; 21: 3295-3313.

6. Shivdasani RA. MicroRNAs: regulators of gene expression and cell differentiation. Blood 2006; 108: 3646-3653.

7. Farh KK, Grimson A, Jan C, Lewis BP, Johnston WK, Lim LP et al. The widespread impact of mammalian MicroRNAs on mRNA repression and evolution. Science 2005; 310 : 1817-1821.

8. Lim LP, Lau NC, Garrett-Engele P, Grimson A, Schelter JM, Castle J et al. Microarray analysis shows that some microRNAs downregulate large numbers of target mRNAs. Nature 2005; 433: 769-773.

9. Krek A, Grun D, Poy MN, Wolf R, Rosenberg L, Epstein EJ et al. Combinatorial microRNA target predictions. Nat Genet 2005; 37: 495-500.

10. Lewis BP, Burge CB, Bartel DP. Conserved seed pairing, often flanked by adenosines, indicates that thousands of human genes are microRNA targets. Cell 2005; 120: 15-20.

11. Bartel DP, Chen CZ. Micromanagers of gene expression: the potentially widespread influence of metazoan microRNAs. Nat Rev Genet 2004; 5: 396-400.

12. Bartel DP. MicroRNAs: genomics, biogenesis, mechanism, and function. Cell 2004; 116 281-297.

13. Stark A, Brennecke J, Bushati N, Russell RB, Cohen SM. Animal MicroRNAs confer robustness to gene expression and have a significant impact on $3^{\prime}$ UTR evolution. Cell 2005; 123: 1133-1146.

14. Arora A, Simpson DA. Individual mRNA expression profiles reveal the effects of specific microRNAs. Genome Biol 2008; 9: R82.

15. Fontana L, Pelosi E, Greco P, Racanicchi S, Testa U, Liuzzi F et al. MicroRNAs 17-5p-20a-106a control monocytopoiesis through AML1 targeting and M-CSF receptor upregulation. Nat Cell Biol 2007; 9: 775-787.

16. Fazi F, Rosa A, Fatica A, Gelmetti V, De Marchis ML, Nervi $C$ et al. A minicircuitry comprised of microRNA-223 and transcription factors NFI-A and C/EBPalpha regulates human granulopoiesis. Cell 2005; 123: 819-831.

17. Rosa A, Ballarino M, Sorrentino A, Sthandier O, De Angelis FG, Marchioni M et al. The interplay between the master transcription factor PU.1 and miR-424 regulates human monocyte/macrophage differentiation. Proc Natl Acad Sci USA 2007; 104: 19849-19854.

18. Lu J, Guo S, Ebert BL, Zhang H, Peng X, Bosco J et al. MicroRNA-mediated control of cell fate in megakaryocyte-erythrocyte progenitors. Dev Cell 2008; 14: 843-853.

19. Ramkissoon SH, Mainwaring LA, Ogasawara Y, Keyvanfar K, McCoy Jr JP. Sloand EM et al. Hematopoietic-specific microRNA expression in human cells. Leuk Res 2006; 30: 643-647.

20. Romania P, Lulli V, Pelosi E, Biffoni M, Peschle C, Marziali G. MicroRNA 155 modulates megakaryopoiesis at progenitor and precursor level by targeting Ets-1 and Meis1 transcription factors. Br J Haematol 2008; 143: 570-580.

21. Georgantas III RW, Hildreth R, Morisot S, Alder J, Liu CG, Heimfeld S et al. CD34+ hematopoietic stem-progenitor cell microRNA expression and function: a circuit diagram of differentiation control. Proc Natl Acad Sci USA 2007; 104: 2750-2755.

22. Andersson LC, von Willebrand E, Jokinen M, Karhi KK, Gahmberg CG. Glycophorin A as an erythroid marker in normal and malignant hematopoiesis. Haematol Blood Transfus 1981; 26: 338-344.

23. Uzan G, Prenant M, Prandini MH, Martin F, Marguerie G. Tissue-specific expression of the platelet GPIlb gene. J Biol Chem 1991; 266: 8932-8939.

24. Wang $X$, Studzinski GP. The requirement for and changing composition of the activating protein-1 transcription factor during differentiation of human leukemia HL60 cells induced by 1,25-dihydroxyvitamin D3. Cancer Res 2006; 66: 4402-4409.

25. Eades-Perner AM, Thompson J, van der Putten H, Zimmermann W. Mice transgenic for the human CGM6 gene express its product, the granulocyte marker CD66b, exclusively in granulocytes. Blood 1998; 91: 663-672.

26. Garzon R, Pichiorri F, Palumbo T, Iuliano R, Cimmino A, Aqeilan R et al. MicroRNA fingerprints during human megakaryocytopoiesis. Proc Natl Acad Sci USA 2006; 103 5078-5083.

27. Felli N, Fontana L, Pelosi E, Botta R, Bonci D, Facchiano F et al. MicroRNAs 221 and 222 inhibit normal erythropoiesis and erythroleukemic cell growth via kit receptor downmodulation. Proc Natl Acad Sci USA 2005; 102: 18081-18086.

28. Choong ML, Yang HH, McNiece I. MicroRNA expression profiling during human cord blood-derived CD34 cell erythropoiesis. Exp Hematol 2007; 35: 551-564.

29. Zhan M, Miller CP, Papayannopoulou T, Stamatoyannopoulos G, Song CZ. MicroRNA expression dynamics during murine and human erythroid differentiation. Exp Hematol 2007; 35: 1015-1025.

30. Huang JC, Babak T, Corson TW, Chua G, Khan S, Gallie BL et al. Using expression profiling data to identify human microRNA targets. Nat Methods 2007; 4: 1045-1049.

31. Chen CZ, Li L, Lodish HF, Bartel DP. MicroRNAs modulate hematopoietic lineage differentiation. Science 2004; 303: 83-86.

32. Montanari M, Gemelli C, Tenedini E, Zanocco Marani T, Vignudelli T, Siena M et al. Correlation between differentiation plasticity and mRNA expression profiling of CD34+derived CD14- and CD14+ human normal myeloid precursors. Cell Death Differ 2005; 12 $1588-1600$.

33. Tenedini E, Fagioli ME, Vianelli N, Tazzari PL, Ricci F, Tagliafico E et al. Gene expression profiling of normal and malignant CD34-derived megakaryocytic cells. Blood 2004; 104: 3126-3135.

34. Grande A, Piovani B, Aiuti A, Ottolenghi S, Mavilio F, Ferrari G. Transcriptional targeting of retroviral vectors to the erythroblastic progeny of transduced hematopoietic stem cells. Blood 1999; 93: 3276-3285.

35. Livak KJ, Schmittgen TD. Analysis of relative gene expression data using real-time quantitative PCR and the 2(-Delta Delta C(T)) Method. Methods 2001; 25: 402-408.

36. Ferrari F, Bortoluzzi S, Coppe A, Basso D, Bicciato S, Zini R et al. Genomic expression during human myelopoiesis. BMC Genomics 2007; 8: 264.

37. Salati S, Zini R, Bianchi E, Testa A, Mavilio F, Manfredini R et al. Role of CD34 antigen in myeloid differentiation of human hematopoietic progenitor cells. Stem Cells 2008; 26: 950-959.

\section{(c)}

OMERा:H

censed under a Creative Commons Attribution-Noncommercial-No Derivative Works 3.0 License. To view a copy of this license, visit http:// creativecommons.org/licenses/by-nc-nd/3.0/

\section{Supplementary Information accompanies the paper on Cell Death and Disease website (http://www.nature.com/cddis)}

